\title{
Transformerless Ultrasonic Ranging System with the Feature of Intrinsic Safety for Explosive Environment
}

\author{
Yu Wang ${ }^{1} \oplus$, Yuheng Qiao ${ }^{2}$, Hongjuan Zhang ${ }^{2}$, Yan Gao ${ }^{2}$, Ming Zhang ${ }^{3}$, Heng Tan ${ }^{2}$, \\ Dong Wang ${ }^{1}$ and Baoquan Jin ${ }^{1, *}$ \\ 1 Key Laboratory of Advanced Transducers and Intelligent Control Systems (Ministry of Education and \\ Shanxi Province), College of Physics and Optoelectronics, Taiyuan University of Technology, Taiyuan 030024, \\ China; wangyu@tyut.edu.cn (Y.W.); wangdong@tyut.edu.cn (D.W.) \\ 2 College of Electrical and Power Engineering, Taiyuan University of Technology, Taiyuan 030024, China; \\ qiaoyuheng0266@link.tyut.edu.cn (Y.Q.); zhanghongjuan@tyut.edu.cn (H.Z.); gaoyan@tyut.edu.cn (Y.G.); \\ tanheng0207@link.tyut.edu.cn (H.T.) \\ 3 Center of Nanosciences and of Nanotechnologies, University of Paris-Saclay, 91405 Orsay, France; \\ ming.zhang@u-psud.fr \\ * Correspondence: jinbaoquan@tyut.edu.cn; Tel.: +86-138-3515-5702
}

Received: 1 November 2018; Accepted: 8 December 2018; Published: 12 December 2018

\begin{abstract}
The transformer used in the conventional ultrasonic ranging system could provide a huge instantaneous driving voltage for the generation of ultrasonic wave, which leads to the safety problem in the explosive mixture. This paper proposes a transformerless ultrasonic ranging system powered by the intrinsically safe power source and analog switches. The analysis of intrinsic characteristics of ultrasonic driving circuit is realized in normal and fault conditions. The echo-processing circuit combined with LIN bus technology is further adopted in order to improve the system stability. After the analysis of the timing diagram of ranging instruction, the evaluation experiments of ranging accuracy and ranging stability are completed. The results show that the system can realize reliable bidirectional communication between the LIN master node circuit and the ultrasonic transceiver unit, which realizes the transformerless driving. The system can realize the distance measurement within the range of $250-2700 \mathrm{~mm}$, and the measurement error is less than $30 \mathrm{~mm}$. The measurement fluctuation is less than $10 \mathrm{~mm}$, which provides a new solution for the ultrasonic ranging system in the potentially explosive atmosphere.
\end{abstract}

Keywords: ultrasound transducer; ultrasonic ranging; intrinsic safety; transformerless driving

\section{Introduction}

Due to the narrow working space in the industrial field, many special function vehicles have hidden hazards in the process of reversing, posing a huge threat to safety production [1]. In order to solve these kinds of problems, the ultrasonic detection technology is thus widely used for monitoring and early warning of safety distances because of its simple structure and fast response [2-6], and could be gradually seen in the potentially explosive atmospheres of gases and vapors.

The traditional ultrasonic ranging system driven by the transformer has the disadvantages of large volume and high cost. In this situation, a large driving voltage is required to drive the primary and secondary coils, and a high energy storage is generated while ensuring the driving performance [7]. These effects could be dangerous in explosive atmospheres, because the ultrasound has been considered to be an ignition source in the international safety regulations such as EN 1127-1 [8]. The ignition point of methane-air mixture is about $650{ }^{\circ} \mathrm{C}$. In Reference [9], it is mentioned that the temperature required to ignite combustibles by hot surface methods is higher (about hundreds of degrees). However, this 
condition is far from being met during the operation of ultrasonic ranging system. Therefore, this paper focuses on the ignition caused by electric sparks. Moreover, Reference [10] even suggested a new safety threshold value of $170 \mathrm{~dB}$ for ultrasound coupled directly to gaseous atmospheres. Actually, in normal or fault conditions, when the energy released in the equipment circuit is higher than the minimum ignition energy of the material in the environment, it may ignite the surrounding dust or gas $[11,12]$. For exemple, the large driving voltage in the driving circuit of ultrasound transducer is an important hazards, because the electric spark may be generated if the system is in fault conditions, such as short circuits.

Therefore, in the environment of blasting dust and explosive gas, electrical and electronic equipment must be designed to meet the requirements of safe production $[13,14]$. For the explosion-proof environment, the explosion-proof design of electrical and electronic equipment often uses the oil immersion type, sand filling type, flameproof enclosure, intrinsically safe type, and encapsulation type to separate the safe area from the non-safe area. Among them, the intrinsically safe equipments are widely used, because they eliminate the explosion-proof casing and other complicated structures, and have many advantages such as light weight, small size, low cost, and best safety.

In this paper, we focus on the intrinsic safety design of ultrasonic ranging circuit, and the effect of ignition source ultrasound is not considered here. The effective driving circuit of the ultrasonic transducer under a small voltage is proposed to improve the energy conversion efficiency. The acoustic impedance matching and electrical impedance matching [15-18] are also used to achieve this goal. At the same time, considering the complicated situation in the potentially explosive atmospheres of gases and vapors, the stability of the entire ranging system is improved by introducing the Local Interconnect Networks (LIN) bus which is widely used in the automobile network [19,20]. In summary, the new approach in this paper is that a transformerless ultrasonic ranging system is proposed and the intrinsic characteristics of ultrasonic driving circuit are realized in normal and fault conditions. The echo-processing circuit combined with LIN bus technology is further adopted in order to improve the system stability.

\section{Intrinsically Safe Driving of an Ultrasonic Transducer}

\subsection{Driving Principle of an Ultrasonic Transducer}

In the ultrasonic ranging system, piezoelectric ceramic transducers are often used to realize real-time acquisition of position information. The work of piezoelectric ceramic transducers is based on the inverse piezoelectric effect of piezoelectric crystals, which convert electrical energy into mechanical energy and then into acoustic energy. Under the action of the ultrasonic transducer system, the piezoelectric wafer is resonated by the signal as its own natural frequency, and the resonant plate that is attached there to emit ultrasonic waves.

The overall conversion efficiency of an ultrasonic transducer system mainly depends on power transmission efficiency, mechanical energy transmission efficiency, and electromechanical conversion efficiency. An important indicator affecting the conversion efficiency of mechanical energy and electrical energy is the electromechanical coupling coefficient of piezoelectric crystal materials. This coefficient represents the ratio of the mechanical energy converted by the piezoelectric crystal to the total energy input by the inverse piezoelectric effect in the ultrasonic driving section, which is closely related to the shape of the element, the crystal composition material, and the cutting direction. Since piezoelectric crystals in ultrasonic ranging systems are mostly developed, such as piezoelectric ceramic transducer (PZT)-based piezoelectric ceramics which have been developed, a space for efficiency improvement of the piezoelectric material itself, especially the electromechanical coupling coefficient, has been very limited. In order to meet the requirements of intrinsically safe driving, it is necessary to optimize the energy matching and mechanical energy matching transmission efficiency to realize 
the driving in the ultrasonic transducer at a small voltage. The internal structure of the ultrasonic transducer is shown in Figure 1.

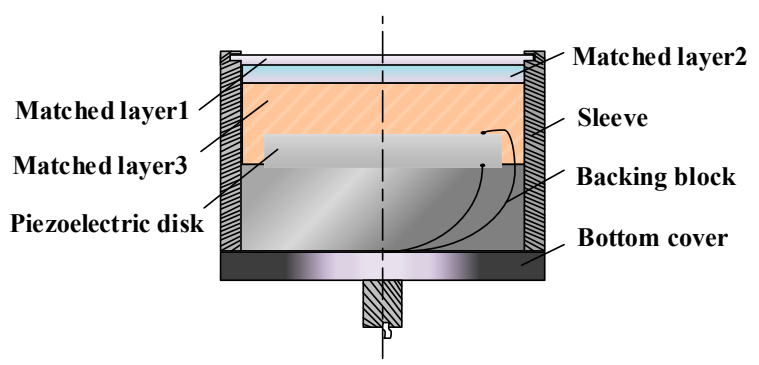

Figure 1. The internal structure of an ultrasonic transducer.

\subsection{Acoustic Impedance Matching of the Ultrasonic Transducer}

The sound waves of the ultrasonic transducer used for ultrasonic ranging are transfered from a solid environment to a gaseous environment. When the sound waves pass through the two media interfaces, they will generate reflected waves and transmitted waves. As shown in Figure 2a, when sound wave is vertically incident from medium 1 to medium 2 , the sound transmittance $t$ and the sound intensity transmittance $T$ satisfy the following conditions [21]:

$$
\begin{gathered}
t=\frac{P_{t}}{P_{0}}=\frac{2 Z_{2}}{Z_{1}+Z_{2}} \\
T=\frac{I_{t}}{I_{0}}=\frac{4 Z_{1} Z_{2}}{\left(Z_{1}+Z_{2}\right)^{2}}
\end{gathered}
$$

where $Z_{1}$ and $Z_{2}$ are the acoustic impedances of media 1 and 2, respectively; $P_{0}$ and $P_{t}$ are the incident and transmitted sound pressures, respectively; and $I_{0}$ and $I_{t}$ are the incident and projected sound intensities, respectively. The acoustic impedance $Z$ is satisfied as following:

$$
Z=\rho c
$$

where $\rho$ is the medium density and $c$ is the wave velocity in the medium. It can be seen from the Equation (3) that the acoustic transmittance at the interface is related to the material itself. In actual use, if the emitting surface is made of aluminum, the sound intensity transmission $T$ is:

$$
T=\frac{I_{t}}{I_{0}}=\frac{4 Z_{1} Z_{2}}{\left(Z_{1}+Z_{2}\right)^{2}}=0.0094 \%
$$

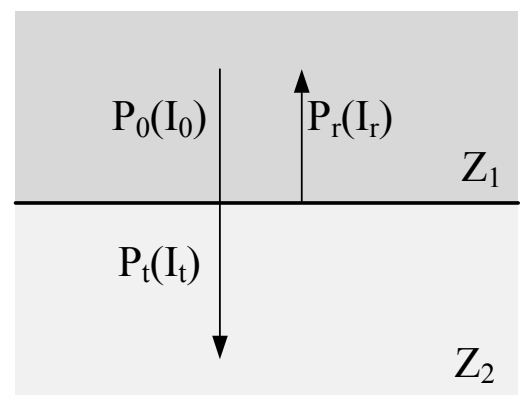

(a)

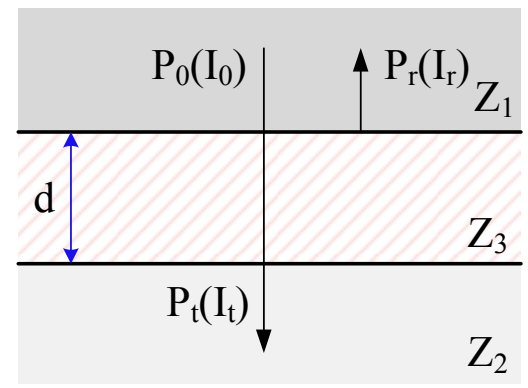

(b)

Figure 2. The schematic of ultrasonic vertical incidence. (a) Acoustic wave incident directly, (b) acoustic wave incident through the matched layer. 
The problem of too low transmission intensity at the time of direct incidence is shown in Figure $2 b$. Improvement is made by adding a matching layer, medium 3, between media 1 and 2 . When the thickness $\mathrm{d}$ of medium 3 satisfies the following equation, there is a maximum sound intensity transmittance $T_{\max }$. The thickness $\mathrm{d}$ of medium 3 and the maximum sound intensity transmittance $T_{\max }$ can be written as:

$$
\begin{gathered}
\mathrm{d}=\frac{\gamma}{4} 2 n-1, n=1,2,3 \ldots \\
T_{\text {max }}=\frac{4 Z_{1} Z_{3}^{2} Z_{2}}{\left(Z_{1} Z_{2}+Z_{3}^{2}\right)^{2}}
\end{gathered}
$$

where $\gamma$ is the wavelength of the sound wave in medium 3 , and $Z_{3}$ is the acoustic impedance of medium 3. When the acoustic impedance $Z_{3}$ of medium 3 satisfies the equation: $Z_{3}=\sqrt{Z_{1} Z_{2}}, T_{\max }$ takes a value of 1 , which means the sound signal does not reflect at all. Therefore, it can be known that the sound transmission efficiency can be improved by reasonably selecting the matching material and the matching layer thickness, thereby improving the overall energy transmission efficiency of the overall driving system.

The mechanical vibration characteristics of ultrasonic piezoelectric transducers can also be expressed in terms of electrical quantities. In order to analyze the load impedance of the transducer, it is necessary to construct a transducer equivalent circuit by matching the appropriate inductor or capacitor to improve the energy conversion efficiency of the transducer. The equivalent circuit of the ultrasonic transducer is shown in Figure 3.

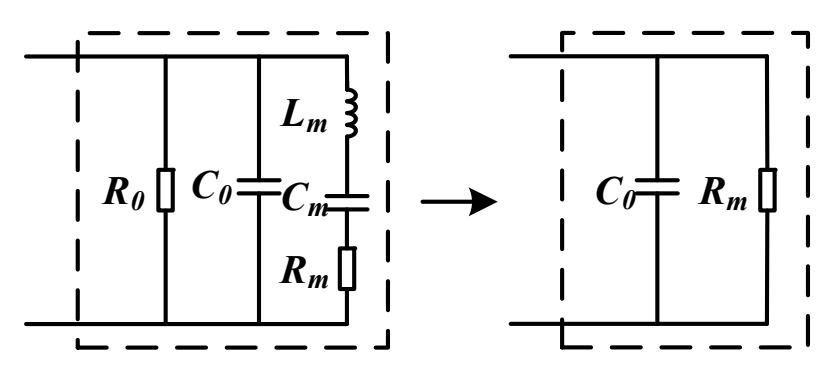

(a)

(b)

Figure 3. Equivalent circuit structure of the ultrasonic transducer: (a) equivalent circuit diagram of the transducer before resonance, (b) equivalent circuit diagram of the transducer after resonance.

Figure 3a is the equivalent circuit diagram of the ultrasonic transducer before resonance, where $C_{0}$ is the static capacitance of the ultrasonic transducer due to clamping, and $R_{0}$ is the dielectric resistance of the ultrasonic transducer. Since $R_{0}$ is considered to be infinite, the influence is generally ignored. $L_{m}, C_{m}$ and $R_{m}$ are the dynamic inductance, capacitance, and resistance of the transducer, respectively. When the frequency of transducer driving pulse is equal to the resonant frequency $f_{s}$ of the ultrasonic transducer, $L_{m}$ and $C_{m}$ undergo series resonance. The series branch is pure resistance $R_{m}$, the equivalent circuit of the transducer is shown in Figure $3 \mathrm{~b}, R_{m}$ and $C_{0}$ are connected in parallel, and the impedance is capacitive. The resonant frequency $f_{s}$ of the ultrasonic transducer is described as:

$$
f_{s}=\frac{1}{\sqrt{L_{m} C_{m}}}
$$

\subsection{Design of the Intrinsically Safe Ultrasonic Driving Circuit}

The core of the ultrasonic transducer system is a layered resonance structure based on piezoelectric materials, which shows the characteristics of capacitive load. Therefore, it is necessary to adopt electrical impedance matching method to improve the output power of the signal source to the transducer during power transmission. In order to realize the transmission of ultrasonic waves, it is necessary to design a pulse driving circuit to generate an electric pulse having the same resonance 
frequency as that of the ultrasonic transducer and having a large driving voltage, and to drive the ultrasonic transducer to vibrate after matching the network, for the purpose of transmitting ultrasonic waves.

The ultrasonic transducer used in this scheme is an anti-drip-type transceiver-integrated ultrasonic probe (model: MA58MF14-0N), which is shown in Figure 4, and its electrical performance parameters are listed in Table 1.

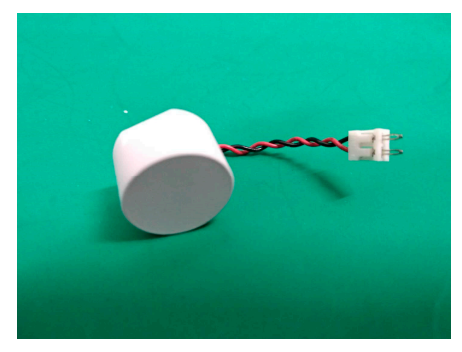

Figure 4. Physical map of the ultrasonic transducer.

Table 1. Electrical parameters of ultrasonic transducer.

\begin{tabular}{cccc}
\hline $\begin{array}{c}\text { Nominal Frequency } f_{s} \\
(\mathbf{k H z})\end{array}$ & $\begin{array}{c}\text { Static Capacitance } C_{\mathbf{0}} \\
(\mathbf{p F})\end{array}$ & $\begin{array}{c}\text { Aftershock Time } T \\
(\mathbf{m s})\end{array}$ & $\begin{array}{c}\text { Maximum Input Voltage } V_{\text {max }} \\
(\mathbf{V p p})\end{array}$ \\
\hline $58 \pm 1.5$ & $1400 \pm 20 \%$ & 1.4 & 120 \\
\hline
\end{tabular}

In order to meet the intrinsically safe driving requirements, a transformerless ultrasonic driving circuit is designed and shown in Figure 5. The circuit consists of the impedance matching and the pulse generation. $R_{1}$ and $C_{3}$ are the matching resistor and the matching capacitor of the driving circuit, respectively. The echo-receiving capacitors $C_{1}$ and $C_{2}$ and the echo-receiving resistors $R_{2}$ and $R_{3}$ are chosen carefully in order to complete the reception of the echo signal.

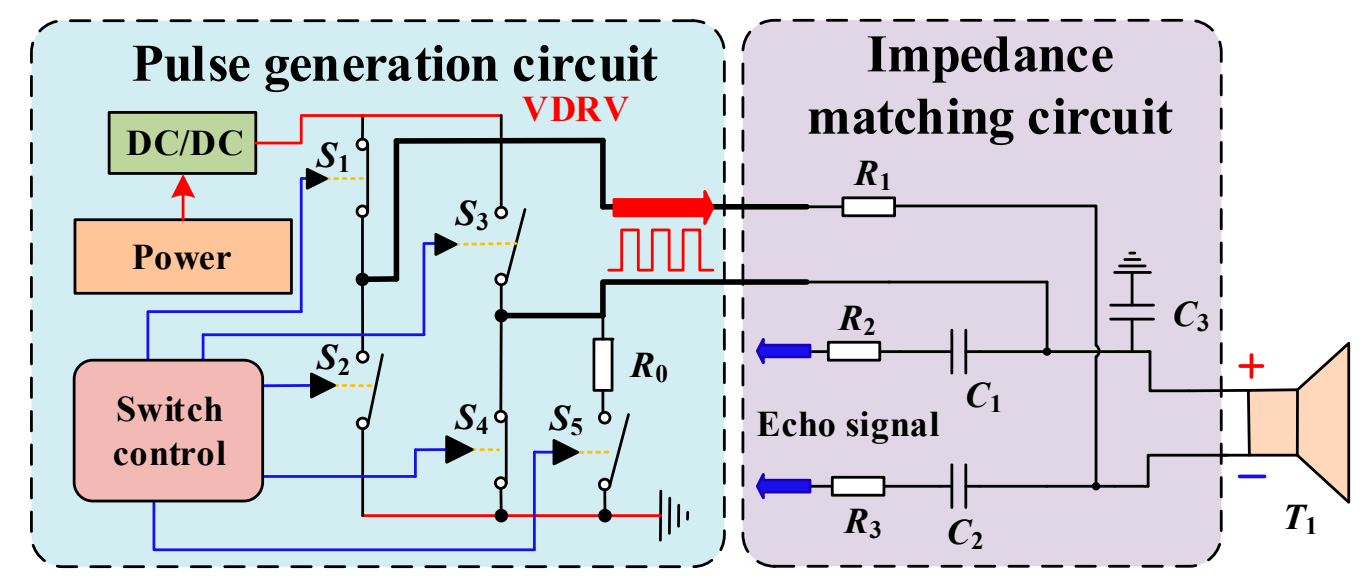

Figure 5. Intrinsically safe ultrasonic driving circuit.

The system is powered by an intrinsically safe power supply $(18 \mathrm{~V})$ and produces the required driving voltage $V_{D R V}(12 \mathrm{~V})$ via a direct current $(\mathrm{DC}) / \mathrm{DC}$ converter. The pulse generation circuit is composed of four analog switches and controllers, i.e., $S_{1}, S_{2}, S_{3}$, and $S_{4}$. When the positive pulse is driven, the switches $S_{2}$ and $S_{3}$ are turned on, and $S_{1}$ and $S_{4}$ are turned off. At this time, the positive voltage of the transducer is $V_{D R V}$, the negative terminal is 0 ; in the negative pulse driving state, the switches $S_{1}$ and $S_{4}$ are turned on, and $S_{2}$ and $S_{3}$ are turned off. At this time, the negative voltage of the transducer is $V_{D R V}$ and the positive terminal is 0 . When the controller controls the switch to turn on and off with the resonant frequency $f_{S}$ of the transducer, a square wave of amplitude $V_{D R V}$ and frequency $f_{s}$ can be generated at both ends of the transducer, thereby ensuring normal operation of the 
transducer. After the end of a set of 16 pulses, the controller controls $S_{0}$ to close, and the damping $R_{0}$ is connected to the circuit to reduce the ultrasonic "tailing" caused by mechanical inertial vibration, for the purpose of reducing the dead zone.

\subsection{Analysis of Intrinsic Characteristics of the Ultrasonic Driving Circuit}

\subsubsection{Analysis of Intrinsic Characteristics of Circuit In Normal Conditions}

In the driving circuit of the ultrasonic ranging circuit, the energy storage component has a large energy storage, so it is difficult to meet the intrinsic safety requirements. Therefore, the capacitor energy storage is the first consideration of the design, and the energy storage of the capacitor is calculated as follows:

$$
\mathrm{Wc}=\frac{1}{2} C U^{2}
$$

where $U$ is the voltage across the capacitor $C$, and in order to meet the intrinsic safety performance requirements of the ultrasonic ranging device, combined with the equivalent circuit model of the ultrasonic transducer and the transformerless ultrasonic driving circuit in Figures 3 and 5, respectively, the circuit model of the driving system shown in Figure 6 is obtained, and the description of each parameter is shown in Table 2. $R_{1}$ is the matching resistor and $C_{3}$ is the matching capacitor. $C_{1}$ and $C_{2}$ are the echo-receiving capacitors. These four designations of the components have the same designations, as those in Figure 5. $C_{0}$ is the static capacitance. $L_{m}, C_{m}$, and $R_{m}$ are the dynamic inductance, capacitance, and resistance of the transducer, respectively. These four designations of the components have the same designations as those in Figure 3. $R_{11}$ and $R_{22}$ are equivalent resistances between the echo-receiving terminal and the ground.

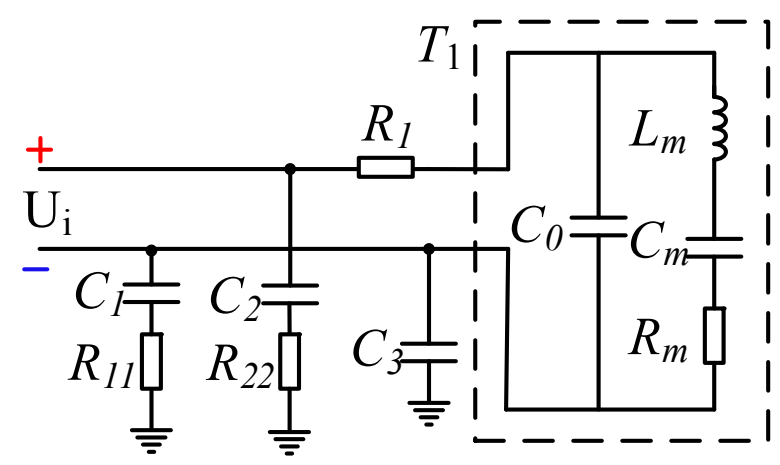

Figure 6. The circuit model of driving system.

Table 2. Circuit parameters of the driving system.

\begin{tabular}{cccc}
\hline $\begin{array}{c}\text { Driving Voltage } \\
\mathbf{U}_{\mathbf{i}}(\mathbf{V})\end{array}$ & $\begin{array}{c}\text { Matching Resistance } R_{\mathbf{1}} \\
(\boldsymbol{\Omega})\end{array}$ & $\begin{array}{c}\text { Matching Capacitance } \\
\boldsymbol{C}_{\mathbf{3}}(\mathrm{pF})\end{array}$ & $\begin{array}{c}\text { Echo-Receiving Capacitance } \\
\boldsymbol{C}_{\mathbf{2}}(\mathrm{pF})\end{array}$ \\
\hline \pm 12 & 100 & 220 & 470 \\
\hline Equivalent Resistance & $\begin{array}{c}\text { Equivalent Resistance } \\
\boldsymbol{R}_{\mathbf{1 1}}(\mathbf{k} \boldsymbol{\Omega})\end{array}$ & $\begin{array}{c}\text { Static Capacitance } \\
\boldsymbol{C}_{\mathbf{0}}(\mathrm{pF})\end{array}$ & $\begin{array}{c}\text { Echo-Receiving Capacitance } \\
\boldsymbol{C}_{\mathbf{1}}(\mathrm{pF})\end{array}$ \\
\hline 100 & 100 & 1400 & 470 \\
\hline
\end{tabular}

The driving pulse is obtained by the switch at a frequency $f$ and the voltage source $V_{D R V}$. When the driving pulse frequency $f$ is equal to the resonance frequency of the ultrasonic transducer, $L_{m}$ and $C_{m}$ undergo series resonance. The series branch is a pure resistor $R_{m}$. In the ultrasonic-distance-measured device, the 16-pulse driving method is usually used, and the voltage between the two ends of the driver $(+,-)$ is obtained as follows:

$$
\mathrm{Ui}+(n)=\frac{V_{D R V}}{2}\left((-1)^{n+1}+1\right)
$$




$$
\begin{aligned}
\mathrm{Ui}-(n) & =\frac{V_{D R V}}{2}\left((-1)^{n}+1\right) \\
\mathrm{Ui}(n) & =V_{D R V}(-1)^{n+1}
\end{aligned}
$$

where $n(n=1,2,3, \ldots, 32)$ is a half-pulse ordinal. Then, during the positive and negative pulses, the equivalent circuit of the driving system is shown in Figure 7. The descriptions of the components in Figure 7 are shown in Table 2 and these element parameters in Figure 7 have the same meanings as those in Figure 6.

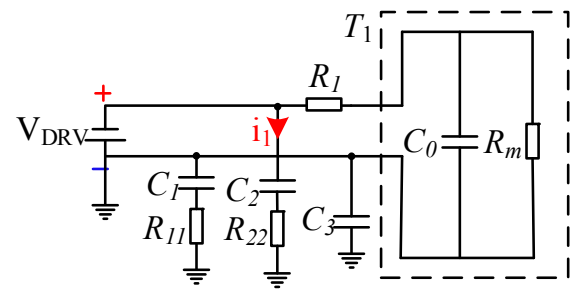

(a)

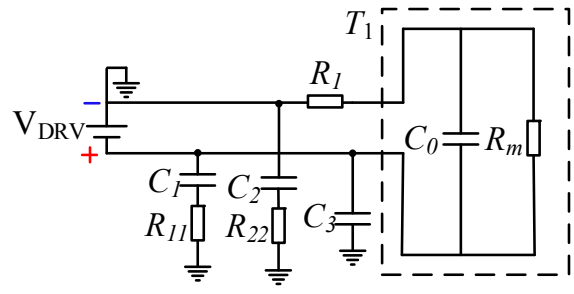

(b)

Figure 7. The equivalent circuit model of different driving states (a) for positive pulse driving and (b) for negative pulse driving.

As shown in Figure 7a, during positive pulse driving, $C_{1}$ and $C_{3}$ are short-circuited to the ground. If $C_{2}$ is stored, its energy is

$$
W_{C_{02}}=\frac{1}{2} C U^{2}=0.5 \times 470 \times 10^{-12} \times 12^{2}=33.84 \times 10^{-9} \mathrm{~J}=33.84 \mathrm{~nJ}
$$

The actual positive pulse duration is $t_{p}$, which is given by:

$$
t_{p}=\frac{1}{2} \times \frac{1}{f}=0.5 \times \frac{1}{58 \times 10^{3}}=8.62 \times 10^{-6} s=8.62 \mu \mathrm{s}
$$

During this period, the path formed by $C_{2}$ and $R_{22}$ satisfies:

$$
R_{22} \times C_{2} \times \frac{\mathrm{d} U_{C_{2}}}{\mathrm{dt}}+U_{C_{2}}=V_{D R V}
$$

Then before the end of the positive pulse,

$$
U_{C_{2}}=V_{D R V}\left(1-e^{\frac{-t}{R_{22} \times C_{2}}}\right)=12 \times\left(1-e^{\frac{-8.62 \times 10^{-6}}{100 \times 10^{3} \times 470 \times 10^{-12}}}\right)=2.0112 \mathrm{~V}
$$

Then, the actual energy is:

$$
W_{C_{2}}=\frac{1}{2} C U^{2}=0.5 \times 470 \times 10^{-12} \times 2.0112^{2}=33.84 \times 10^{-9} \mathrm{~J}=0.95 \mathrm{~nJ}
$$

According to the national and international standards, in the potentially explosive atmospheres of gases and vapors with class I (methane-air), the minimum ignition energy $W_{\min }=0.28 \mathrm{~mJ}[22,23]$, according to the above results $W_{C_{2}} \ll W_{C_{02}} \ll W_{\text {min }}$, so the selection of capacitor $C_{2}$ meets the intrinsic safety requirements.

Similarly, as shown in Figure $8 \mathrm{~b}$, during the negative pulse driving, $C_{2}$ is short-circuited to the ground. If $C_{1}$ and $C_{3}$ are stored, their respective energies are given by:

$$
W_{C_{01}}=\frac{1}{2} C U^{2}=0.5 \times 470 \times 10^{-12} \times 12^{2}=33.84 \times 10^{-9} J=33.84 n J
$$




$$
W_{C_{03}}=\frac{1}{2} C U^{2}=0.5 \times 220 \times 10^{-12} \times 12^{2}=15.84 \times 10^{-9} J=15.84 n J
$$

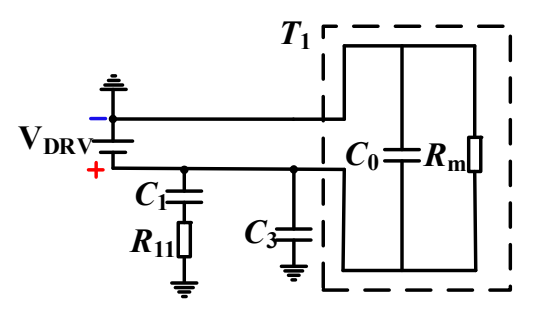

(a)

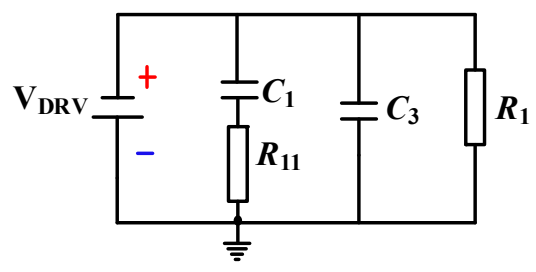

(b)

Figure 8. System equivalent circuit diagrams for the conditions of (a) short circuit at $R_{1}$ and (b) short circuit at $T_{1}$.

According to the above calculation results, $W_{C_{01}} \ll W_{\text {min }}$, and $W_{C_{03}} \ll W_{\text {min }}$, so the capacitors $C_{1}$ and $C_{3}$ are selected to meet the intrinsic safety requirements.

\subsubsection{Analysis of Intrinsic Characteristics of Circuit in Fault Conditions}

Considering that short-circuit faults may be relatively difficult to happen in the internal circuit of the ultrasonic transducer, the conditions of the short circuit of the matching resistor $R_{1}$ and the short-circuit of the transducer $T_{1}$ are analyzed. The equivalent circuit is shown in Figure 8. The descriptions of the components in Figure 8 are shown in Table 2 and these element parameters in Figure 8 have the same meanings as those in Figure 6.

When the short circuit of $R_{1}$ occurs, the negative pulse driving mode is more dangerous than the positive pulse driving. Therefore, as shown in Figure 8a, the system is a capacitive circuit, and the safety factor of the power supply voltage should be 1.5 according to the book by Zhang and Li [24]. Then, the corrected voltage is $1.5 V_{D R V}=18 \mathrm{~V}$, and the maximum equivalent capacitance of the system is $C_{e_{1}}=C_{1}+C_{3}+C_{0}=2090 \mathrm{pF}$. It is checked according to the minimum ignition curve $(C+0 \Omega)$ of the class I capacitive circuit, at $20 \mathrm{~V}$ (greater than $18 \mathrm{~V}$ ), and the corresponding capacitance value is $C_{\min 1},\left(20 \mu \mathrm{F}<C_{\min 1}<30 \mu \mathrm{F}\right)$, that is, $C_{e_{1}} \ll C_{\min 1}$, so the intrinsic safety requirement is also satisfied at this time.

Similarly, as shown in Figure $8 \mathrm{~b}$, when the short circuit of the transducer $T_{1}$ occurs, the system is a capacitive circuit. According to the standard, the safety factor of the power supply voltage should be 1.5 , and the corrected voltage is $1.5 V_{D R V}=18 \mathrm{~V}$. The equivalent capacitance is $C_{e_{2}}=C_{1}+C_{3}=690 \mathrm{pF}$, so it is checked according to the minimum ignition curve $(C+40 \Omega)$ of the class I capacitive circuit. At $20 \mathrm{~V}$ (greater than $18 \mathrm{~V}$ ), the corresponding capacitance value is $C_{\min 2}\left(C_{\min 2} \gg 100 \mu \mathrm{F}\right.$ ), that is, $C_{e_{2}} \ll C_{\min 2}$, so it also meets the requirements of intrinsic safety.

\section{Design of Ultrasonic Echo Signal Processing Based on LIN Bus Technology}

\subsection{LIN Bus and Its Network Structure}

In order to realize the communication and parameter setting of the microcontroller in the ultrasonic transceiver unit, the scheme adopts the LIN bus transceiver chip TJA1020. LIN is a serial communication protocol based on the Universal Asynchronous Receiver/Transmitter (UART)/Serial Communication Interface (SCI) interface, which can be used in various fields such as automobiles and home appliances. As shown in Figure 9, the LIN network as a whole adopts a single bus mode, and the network will enter the running mode after the initialization process is completed; when the sleep command is received or the bus is silent for $4-10 \mathrm{~s}$, the mode will be going into sleep mode, and the network will enter initialization mode by wake-up signals or internal causes. The function of the LIN network is implemented by different nodes such as the master node and the slaves. The node application layer transmits signals and messages downwards through the protocol layer and the 
physical layer, and it can expand up to 16 nodes in an LIN network. The master node in the LIN network can not only control the slaves in the network, detect and adjust the bus status, but also communicate with the upper layer network (such as Controller Area Network (CAN)), which has both master and slave tasks, while the slave node only contains slave tasks.

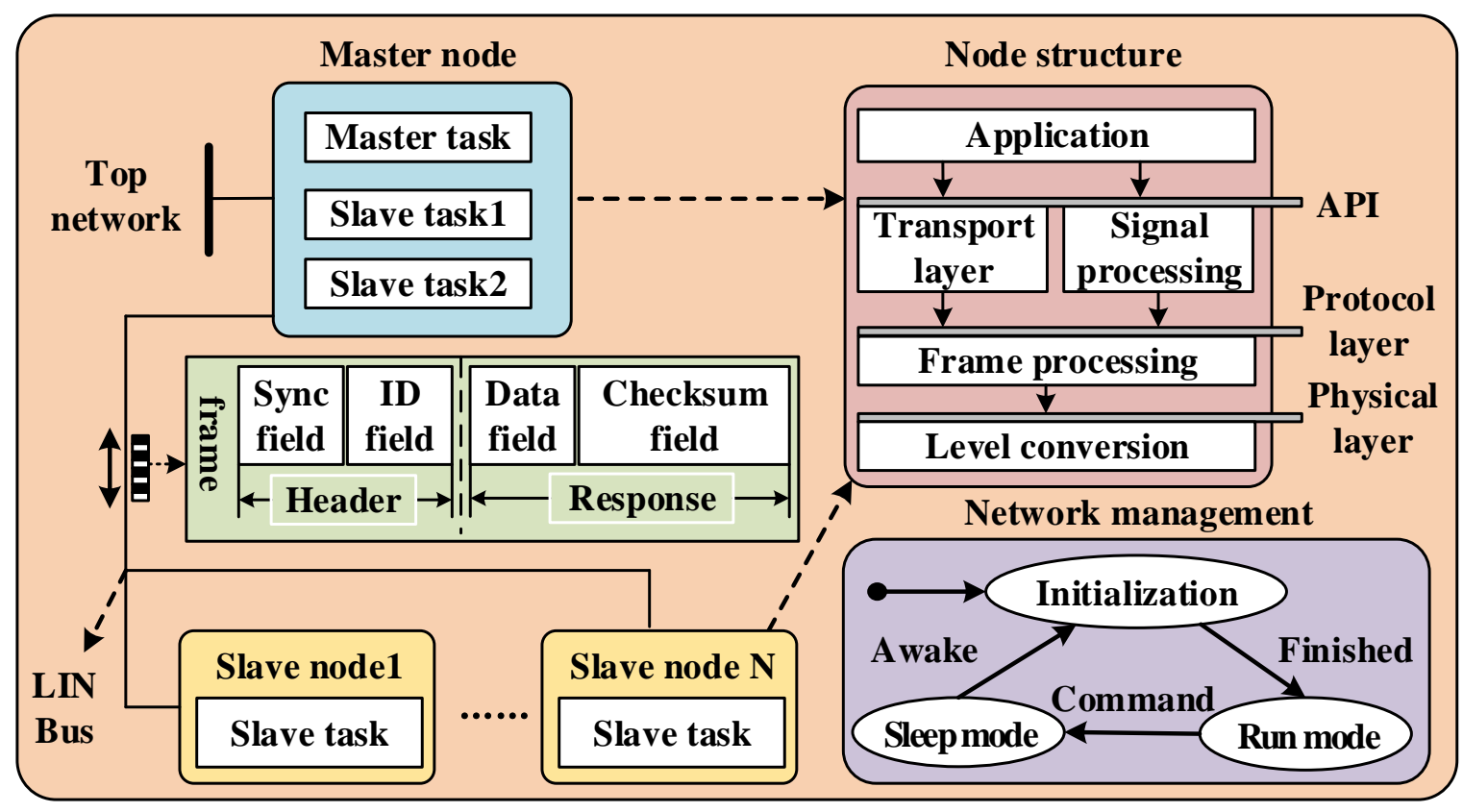

Figure 9. The diagram of Local Interconnect Network (LIN) network and node composition.

The communication message frame format on the LIN bus is fixed, and the bus communication is controlled by the master node. After the master node passes the interval signal, the master node sends the synchronization field and the identifier field as the header of the message, and the slave returns the data field and the checksum field response host. Due to the physical layer limitation, the LIN bus transmission bit rate is up to $20 \mathrm{kbps}$, and the following conditions should be met during communication:

$$
\begin{gathered}
T_{\text {Header }}=34 \cdot T_{\text {bit }} \\
T_{\text {Response }}=\left(10 \cdot N_{\text {Data }}+10\right) \cdot T_{\text {bit }}
\end{gathered}
$$

where $T_{\text {Header }}$ is the nominal transmission time of the header, $T_{\text {bit }}$ is the time required for the primary node to transmit 1 bit of data, $T_{\text {Response }}$ is the response-rated transmission time, and $N_{\text {Data }}$ is the number of bytes contained in the data field. At the same time, in order to ensure the transmission, both $T_{\text {Header }}$ and the $T_{\text {Response }}$ need to leave a certain margin, and the maximum transmission time is 1.4 times the rated transmission time.

\subsection{Design of the Ultrasonic Echo Signal Processing Circuit}

The system echo-processing flow is shown in Figure 10. When the ultrasonic wave is affected by the obstacle and the echo signal is generated, the piezoelectric crystal in the ultrasonic transducer is forced to vibrate to convert the sound signal into a weak electrical signal. The weak electrical signal is amplified by the programmable amplification module and converted into a digital voltage signal through Analog to Digital (A/D) conversion. The digital filter is used to filter out clutter outside $58 \mathrm{kHz}$ to reduce its interference to the system. The filtered signal passes through the echo threshold comparison module. When it is greater than the given value, it is the effective timing termination point. In the process of echo-processing, since the calculation of the obstacle distance time point is generated by voltage threshold comparison, the setting of the reference value for echo comparison is crucial. 


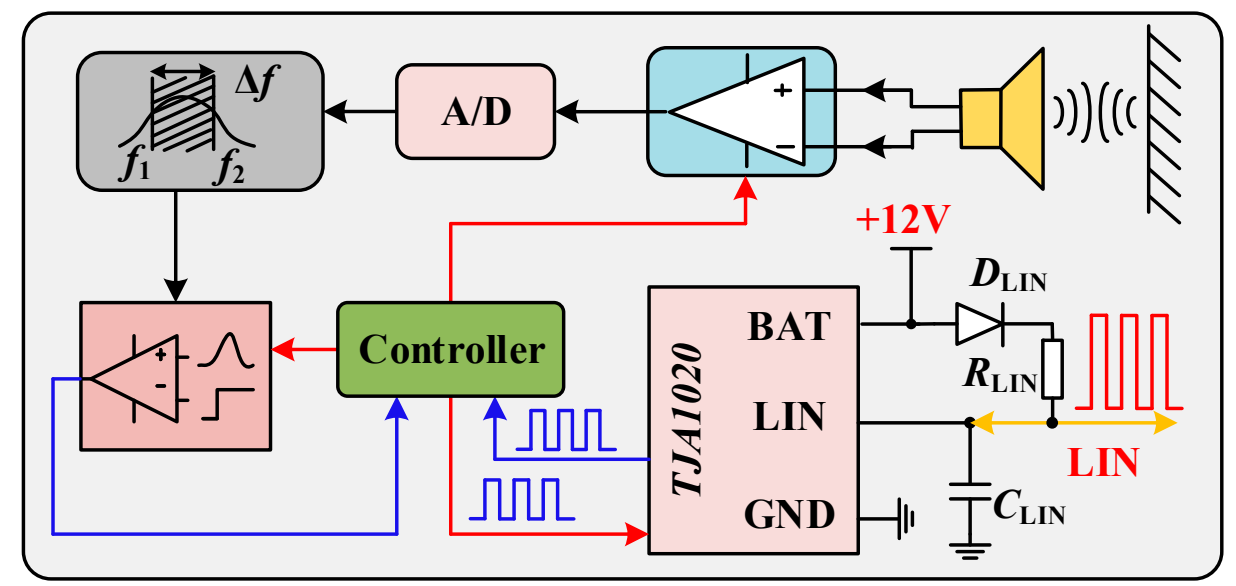

Figure 10. Echo-processing circuit.

In order to improve the stability and anti-interference ability of the echo-processing system, the reverse current diode $D_{\mathrm{LIN}}$ and the LIN matching resistor $R_{\mathrm{LIN}}$ are connected in series between the LIN and Battery supply (BAT) pins of the module. The LIN node capacitor $C_{\mathrm{LIN}}$ is used to control the voltage fluctuation in the LIN. Moreover, the TJA1020-enabled host application mode is used to receive the master controller's instructions and return echo information, as shown in Figure 10. The main controller communicate with the echo controller through LIN to control parameters such as amplification gain and echo comparison threshold. The echo controller returns the threshold comparison result and current status information.

\subsection{LIN Bus Communication Performance Optimization}

In order to ensure the bidirectional operation of the LIN communication to receive the signal fed back by the ultrasonic transceiver unit, the LIN bus voltage should satisfy the threshold condition.

At the dominant level,

$$
V_{\text {LIN_L_MAX }}<0.4 \cdot V_{\text {BAT }}=4.8 \mathrm{~V}
$$

At the recessive level,

$$
V_{\text {LIN_H_MIN }}<0.6 \cdot V_{\text {BAT }}=7.2 \mathrm{~V}
$$

where $V_{\text {LIN_L_MAX }}$ is the highest voltage at a low level in LIN bus; $V_{\text {LIN_H_MIN }}$ is the minimum voltage at a high level in LIN bus; $V_{\mathrm{BAT}}$ is Chip BAT terminal voltage and equal to $12 \mathrm{~V}$, and thus the size of $R_{\mathrm{LIN}}$ needs to be adjusted during the actual process to change the driving capacity of the circuit, so as to meet the requirements in the potentially explosive atmospheres of gases and vapors and implement effective data communication.

\section{Realization of the Transformerless Ultrasonic Ranging System}

\subsection{System Principle Overview}

The intrinsically safe transformerless ultrasonic ranging system is shown in Figure 11, and is mainly composed of a power supply unit, a control unit, a channel selection unit, an LIN communication unit, an ultrasonic transceiver unit, an alarm and calibration unit, and a display unit. The system uses STC12C5A32S2 microcontroller as the core of the control unit. The power supply unit is supplied with $18 \mathrm{~V}$ voltage from the intrinsically safe power supply (model: CSTI-I). The DC/DC conversion chip 78M05 provides the $5 \mathrm{~V}$ voltage required by the system and the MAX5025 generates $12 \mathrm{~V}$ voltage to ensure LIN communication. The channel selection unit realizes switching of the output signal of the control unit, and the LIN communication unit completes conversion of the Transistor-Transistor Logic (TTL) level and the LIN level to realize state control and data transmission of the ultrasonic transceiver unit with the e524.06 chip as the core. 


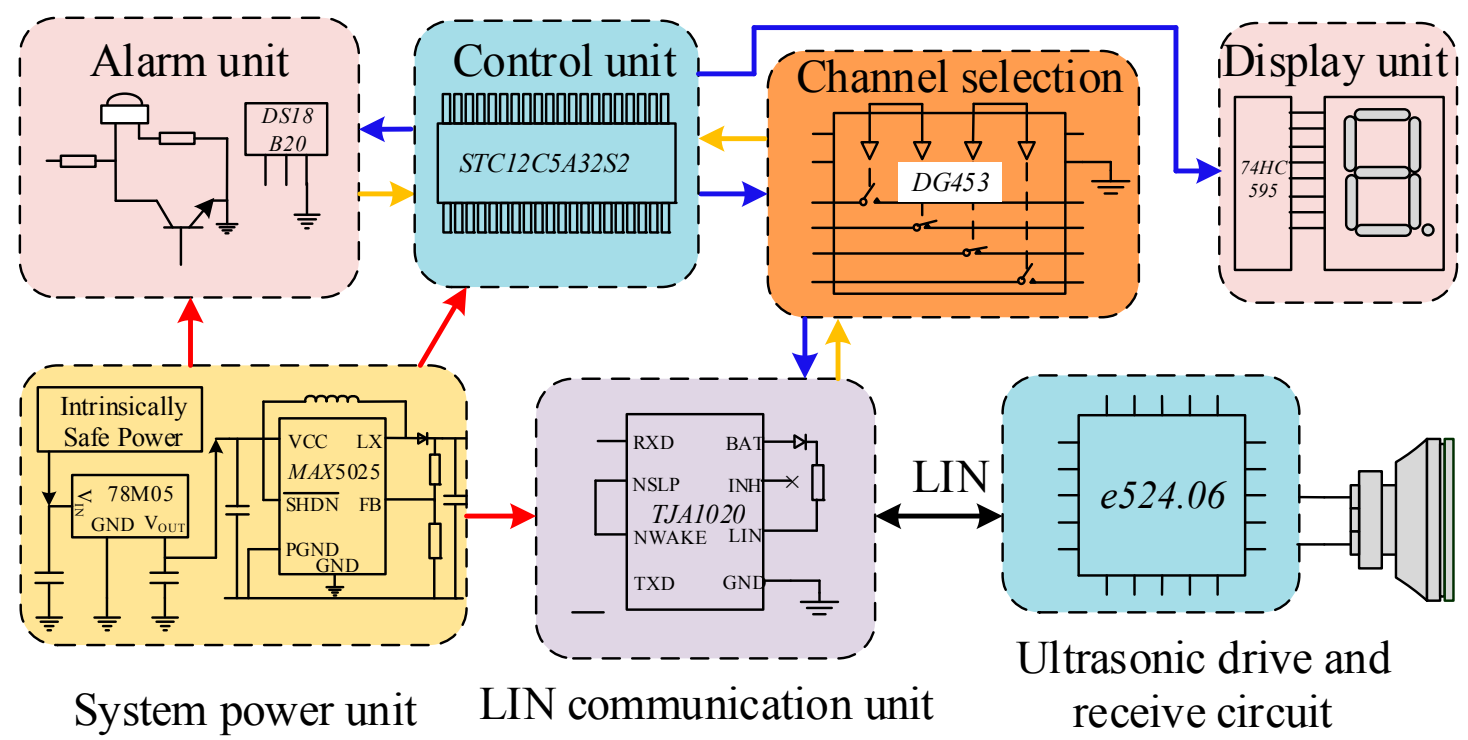

Figure 11. Block diagram of the ultrasonic ranging system.

\subsection{Design of e524.06 Chip Peripheral Circuit}

The potentially explosive atmospheres of gases and vapors are complex and therefore put high demands on the circuit so the system uses the ultrasonic-distance-measuring chip e524.06 which was produced by an elmo company, and designed its peripheral circuit (as shown in Figure 12) to achieve transformerless driving and echo-processing of the ultrasonic transducer. The chip is internally composed of a power module, a transducer driving module, an echo-receiving and -processing module, a clock module, a Joint Test Action Group (JTAG) interface module, a controller module, an Electrically Erasable Programmable Read Only Memory (EEPROM) module, and an Input/Output (I/O) interface module. By modifying the EEPROM configuration information in the chip, not only the adjustment of the ultrasonic transducer driving voltage and frequency, but also the working state of the transducer can be changed, and the static and dynamic modes can be selected to generate the threshold. When static generation is selected, the relevant registers are configured to realize the setting of key parameters, such as linear interpolation, voltage offset and scale factor, to adjust the ultrasonic echo-processing effect to improve the system measurement accuracy.

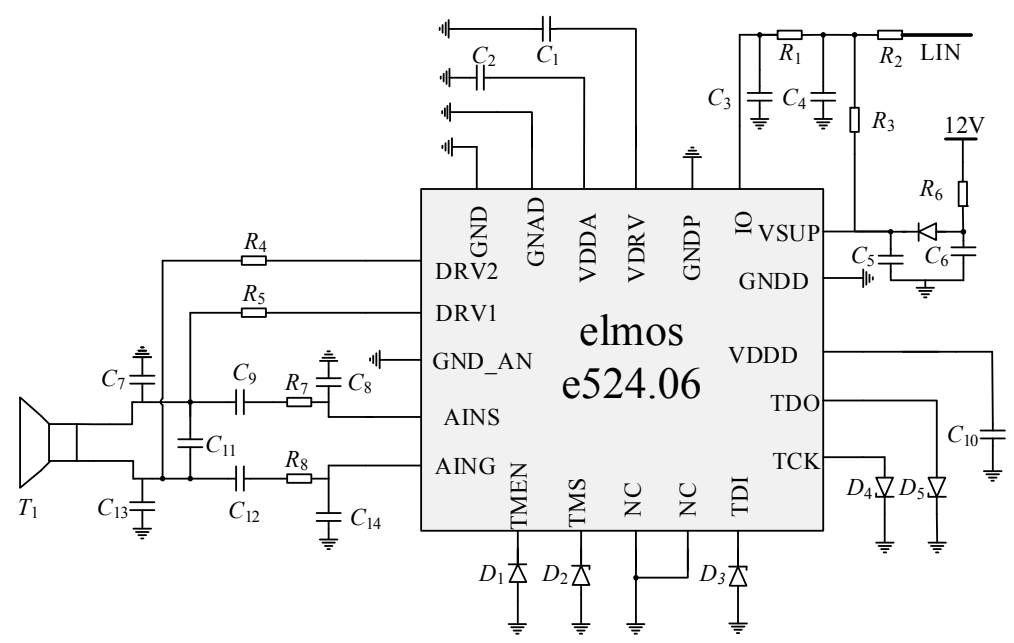

Figure 12. Ultrasonic transmitting and receiving circuit diagram. 


\subsection{Instruction and Ranging Timing of e524.06 Chip}

Writing specific instruction steps are required to realize communication between the microprocessor and e524.06 chip and other functions such as 16-pulse driving wave transmission and EEPROM programming. On the basis of completing the hardware design and compiling the signal path of the ultrasonic ranging system, the calculation of actual distance data is completed according to distance order sequence in Figure 13. The real-time voltage of LIN bus in the ranging process is shown in Figure 13. The system sends ranging instructions and waits for the respond of ultrasonic transceiver between a and b (a range of valid time intervals is 77.6-154 $\mu \mathrm{s}$ ), e524.06 chip in the ultrasonic transceiver evaluates the environmental noise in the measurement process between $\mathrm{c}$ and $\mathrm{d}$, and the length of time $\mathrm{T}$ is the time used for ultrasonic transmission during a single measurement of the system.

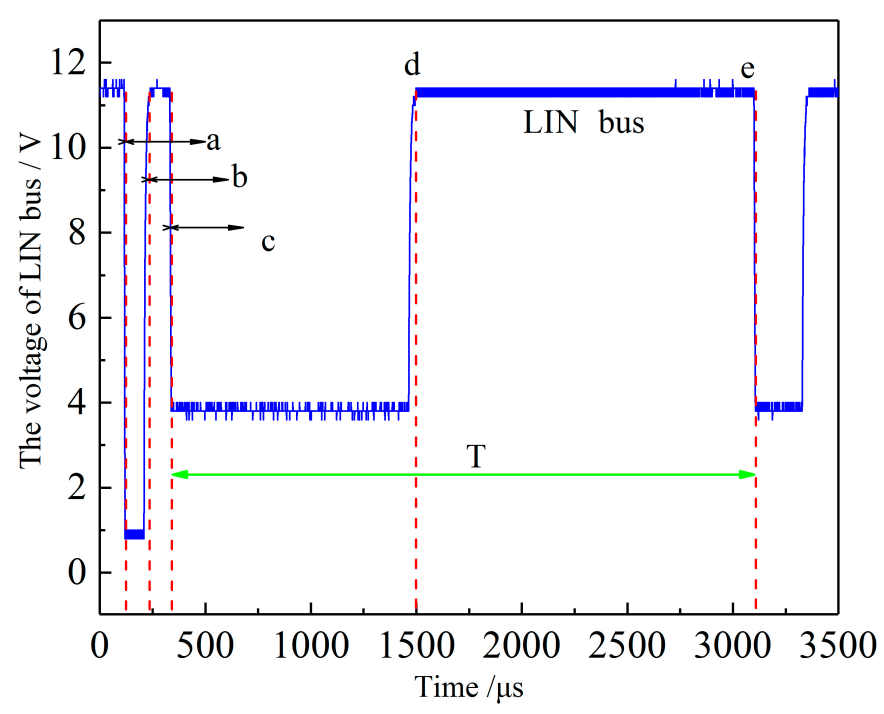

Figure 13. Timing diagram of the ranging command.

Software flow design of the whole ultrasonic ranging system can be realized according to the ranging distance sequence above. The controller issues a distance measurement command (the dominant level between 77.6 and $154 \mu \mathrm{s}$ ) after completing the system initialization, and starts the hardware count function, which calculates ultrasonic wave spreading time according to the difference between the counts of the two falling edges and system command cycle, with the help of programmable counter array (PCA) edge capture function of single-chip microcomputer (STC12C32S2).

\subsection{Software Flow of the System Ranging Process}

The working flow chart of the ranging system is shown in Figure 14. The system waits for the falling edge of $c$ time (waiting for interrupt) from time $b$. When it enters the interrupt program, the system takes out the current timing value and uses it as the starting point of echo time. The system waits for the next falling edge trigger (e time) after processing the above, taking the current count value in the interrupt program as the end point of the echo time, clears the counter and related variables, and ends the ranging.

When the ranging is over, the system enters the communication and display module. Firstly, it turns off the capture function of the PCA module, uses the DS18b20 to get the current temperature, and further obtains the sound speed after calibration, and then calculates the current distance based on this. When data transmission is required, the system selects different transmission modes (control mode or acquisition mode), selects channels (single channel or quad channels), and then performs data checksum calculation. On this basis, it is packaged into data frames and sent via the LIN bus. When 
data transmission is not required, the real-time distance display is directly performed and an audible and visual alarm is performed according to the defined safety value.

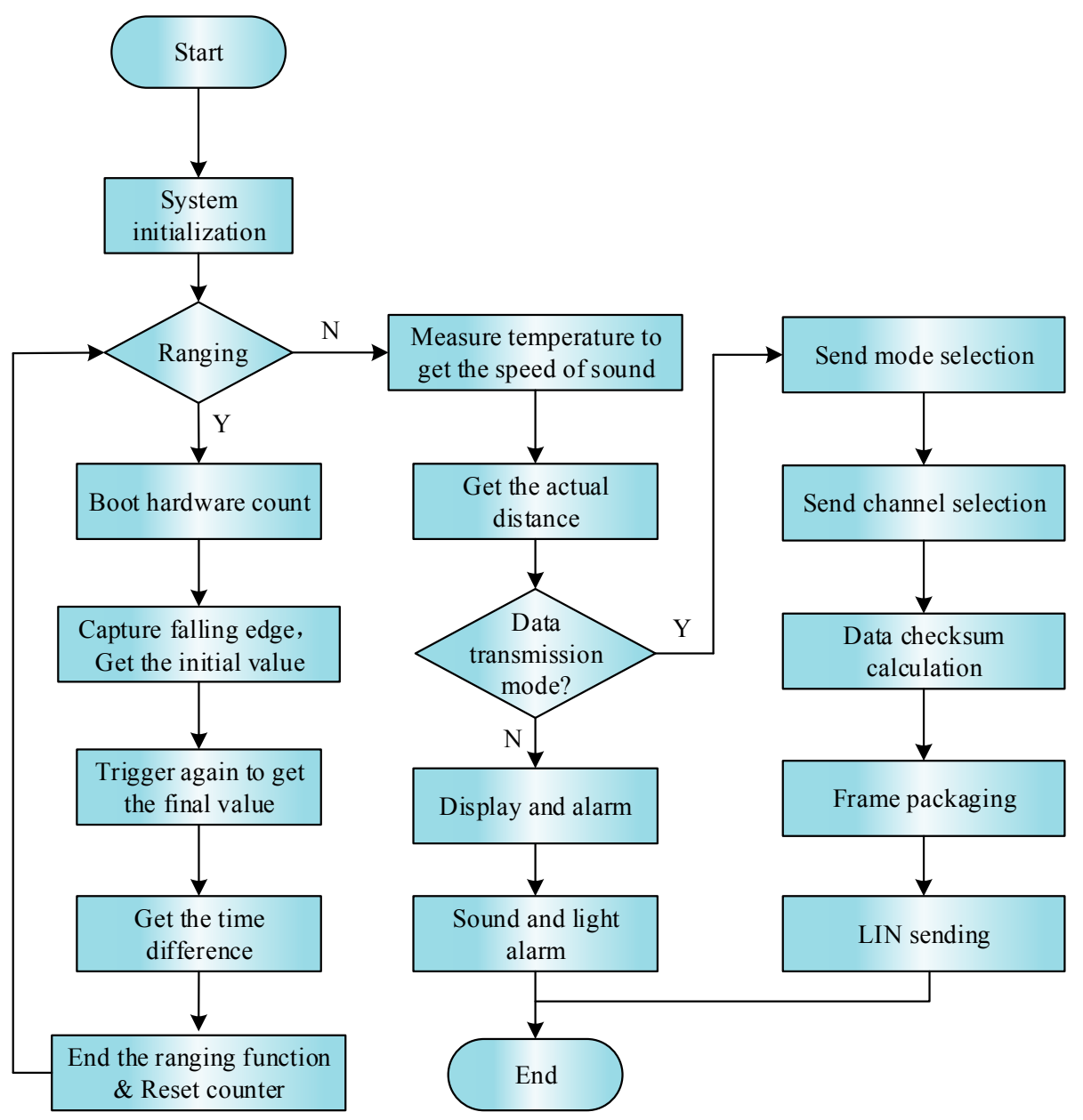

Figure 14. The diagram of system ranging process.

\section{Ultrasonic Ranging Experiment and Analysis}

The level conversion and ranging test system are built to verify the analysis of ultrasonic driving system and LIN bus level part and evaluate the overall function and safety of the system.

\subsection{Analysis of LIN-Bus-Matching Resistance Experiment}

As shown in Figure 15, it is noted that the controlling unit can realize the transmission of ultrasonic ranging instruction when the master node circuit $R_{\mathrm{LIN}}$ is $1 \mathrm{k} \Omega$ during the experiment. However, the controlling unit cannot receive the effective response of the ultrasonic transceiver, which is shown in Figure 15.

The two curves in Figure 15 are the level changes of the LIN bus and the receive data (RXD) port of the TJA1020 chip when the resistance $R_{\mathrm{LIN}}$ in Figure 10 is $1 \mathrm{k} \Omega$, and the controller performs the ranging instruction (before $\mathrm{c}$ ). The RXD terminal voltage varies with the LIN bus voltage (the dominant level is $115 \mu \mathrm{s})$, but at the time $\mathrm{c}$ and e, although the ultrasonic transceiver unit replies to the ranging information and the LIN bus generates a level change, the RXD terminal does not. The corresponding reaction is due to the fact that the "low" level of the LIN bus reaches $5.8 \mathrm{~V}$, which exceeds the decision threshold of the TJA1020 for the highest level of the low level, so the hardware circuit needs to be improved. 


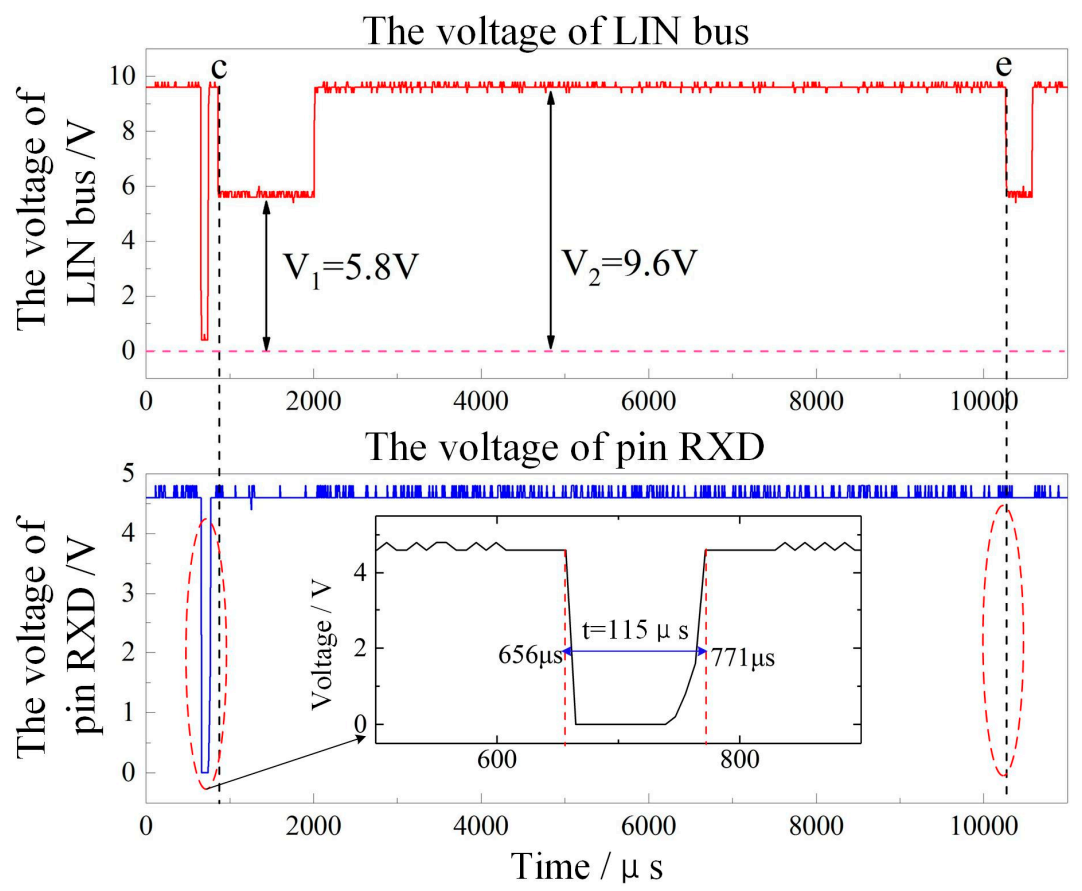

Figure 15. Diagram of the level on LIN communication $\left(R_{\mathrm{LIN}}=1 \mathrm{k} \Omega\right)$.

Table 3 shows the communication level results when the $R_{\text {LIN }}$ takes the values of $1 \mathrm{k} \Omega, 2 \mathrm{k} \Omega$, and $3.5 \mathrm{k} \Omega$. At this time, the ranging command transmission $(123 \mu \mathrm{s}$, and $128 \mu \mathrm{s}$ at the dominant level) can be realized. At point $\mathrm{c}$ and point $\mathrm{e}$, as the voltage of the LIN bus drops, the voltage of RXD also changes. The corresponding low-level potentials of the LIN bus are $4.0 \mathrm{~V}$ and $3.2 \mathrm{~V}$, respectively, in line with the LIN bus low level. The specification, therefore, produces a low level on the RXD side. The experiment also proves that when $R_{\mathrm{LIN}}$ are $2 \mathrm{k} \Omega$ and $3.5 \mathrm{k} \Omega$, the circuit can complete normal communication and data interaction, and realize effective communication between the ultrasonic transceiver unit and the controlling unit.

Table 3. Communication capability test result of variable resistances.

\begin{tabular}{ccccc}
\hline $\begin{array}{c}\text { LIN-Matching Resistor } \\
\boldsymbol{R}_{\text {LIN }}(\mathbf{k} \Omega)\end{array}$ & $\begin{array}{c}\text { LIN High Level } \\
\boldsymbol{V}_{\mathbf{2}}(\mathbf{V})\end{array}$ & $\begin{array}{c}\text { LIN LowLevel } \boldsymbol{V}_{\mathbf{1}} \\
(\mathbf{V})\end{array}$ & $\begin{array}{c}\text { Command Time } t \\
(\boldsymbol{\mu})\end{array}$ & $\begin{array}{c}\text { RX } \\
\text { Effectiveness }\end{array}$ \\
\hline 1 & 9.6 & 5.8 & 115 & Invalid \\
2 & 8.6 & 4.0 & 123 & Valid \\
3.5 & 7.8 & 3.2 & 128 & Valid \\
\hline
\end{tabular}

\subsection{Analysis of Ultrasonic Ranging Instructions and Driving Wave Experiments}

In order to verify the validity of the ranging command of the communication control system composed of the system microprocessor and TJA2010 for the ultrasonic driving system, the results shown in Figure 16 can be obtained by monitoring the LIN command level and the voltage across the transducer. As shown in Figure 16a, at the time $T_{1}$ and $T_{2}$ after the ranging command is issued, a square wave is generated immediately at both ends of the transducer to drive the transducer. As shown in Figure 16b, a total of 16 sets of pulses are generated during the period $T_{11}-T_{12}$ of driving the transducer, and the frequency $f$ is written as:

$$
f=\frac{n}{T_{12}-T_{11}}=\frac{16}{2.74599 \times 10^{-4}-(-1.40043) \times 10^{-6}}=57.97 \mathrm{kHz}
$$




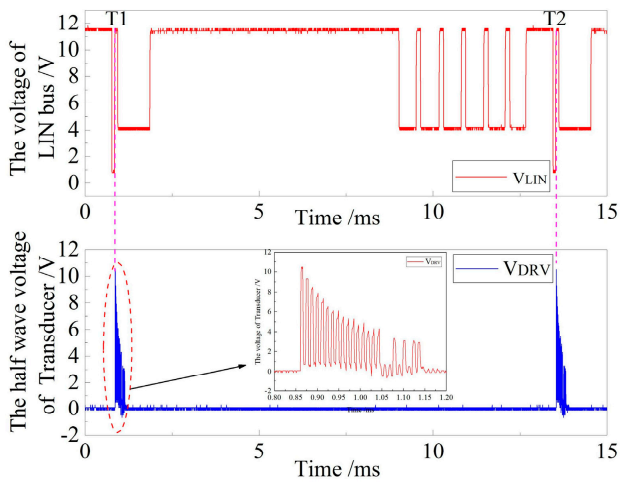

(a)

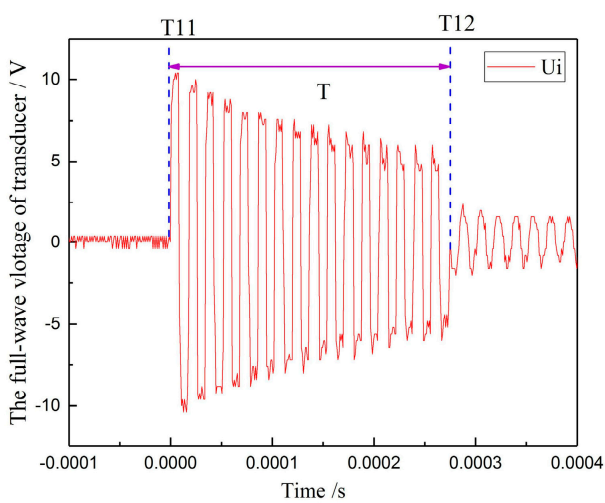

(b)

Figure 16. The diagram of ranging command and transducer voltage: (a) the voltage of LIN bus and half-wave voltage of transducer, and (b) the full-wave voltage of transducer.

\subsection{Analysis of the System Measuring Range}

In the laboratory environment, a $30 \mathrm{~cm} \times 30 \mathrm{~cm} \times 5 \mathrm{~mm}$ iron plate and a 3-m-long guide rail and bracket were used to build a mining ultrasonic ranging experimental platform to measure the range and accuracy of the system. The distance measurement in the range from 240 to $2800 \mathrm{~mm}$ was performed in steps of $100 \mathrm{~mm}$, and the results obtained are shown in Figure 17. The experiment proves that the system can measure the distance measurement within $250 \mathrm{~mm}$ to $2700 \mathrm{~mm}$, and the measurement error increases when the measured distance is closer (within $800 \mathrm{~mm}$ ) and farther (outside $2100 \mathrm{~mm}$ ). When the distance is medium $(900 \mathrm{~mm}-2000 \mathrm{~mm}$ ), the error is smaller and the overall system error is less than $3 \mathrm{~cm}$.

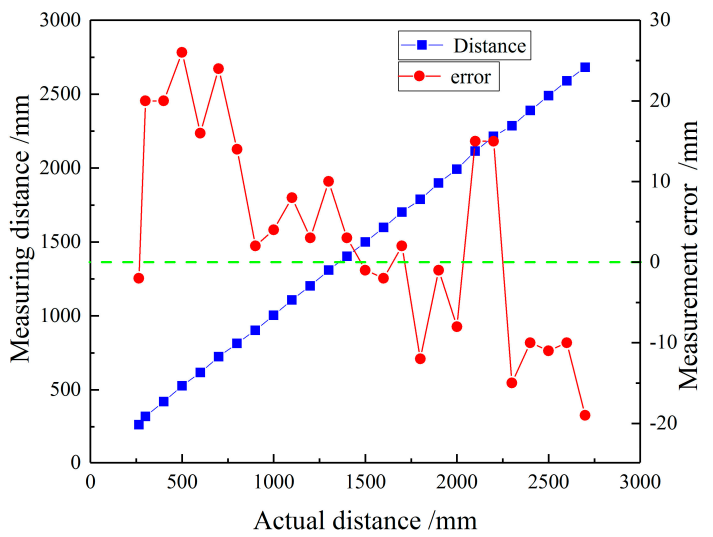

Figure 17. Diagram of the system's measuring rang and error.

At the same time, in order to determine the maximum and minimum values of the system measurement, the experiment was carried out in steps of $5 \mathrm{~mm}$ in the range of $230-260 \mathrm{~mm}$ and 2650-2750 $\mathrm{mm}$, and $30 \mathrm{~mm}$ was the maximum allowable error limit. The experiment results show that the error would be larger if the distance is less than $245 \mathrm{~mm}$ and more than $2705 \mathrm{~mm}$, thus determining the overall measuring range of the system from $250 \mathrm{~mm}$ to $2700 \mathrm{~mm}$.

\subsection{Analysis of System Measuring Stability}

Considering that the system will make larger errors due to other factors such as the external environment in a single measurement, it is necessary to count the multiple measurement results of the system at the same distance to evaluate the stability of the system. Figure 18 shows the measurement results obtained after 50 times of measurements of the system at $500 \mathrm{~mm}, 1200 \mathrm{~mm}, 1600 \mathrm{~mm}$ and $2700 \mathrm{~mm}$. 

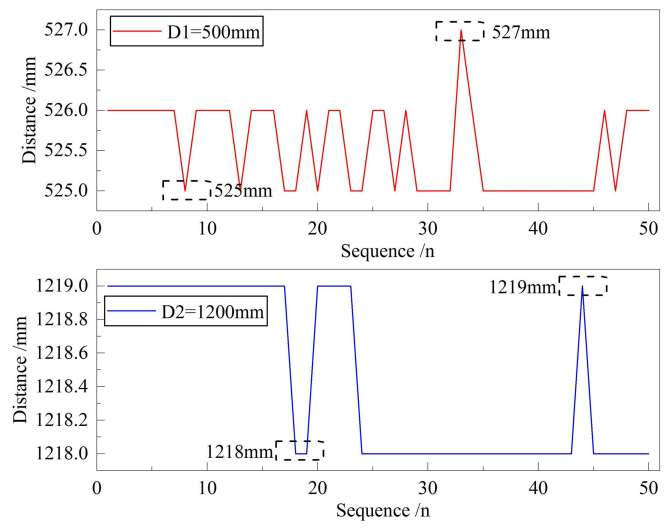

(a)
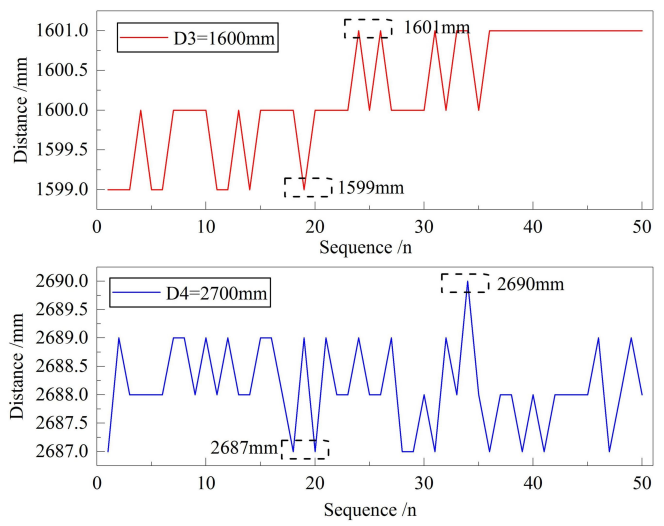

(b)

Figure 18. Diagram of system's measuring stability test: (a) the distances of $500 \mathrm{~mm}$ and $1200 \mathrm{~mm}$, and (b) the distances of $1600 \mathrm{~mm}$ and $2700 \mathrm{~mm}$.

The experimental results show that when the obstacle distances are $500 \mathrm{~mm}, 1200 \mathrm{~mm}, 1600 \mathrm{~mm}$ and $2700 \mathrm{~mm}$, the measurement fluctuations of the ultrasonic ranging system are $2 \mathrm{~mm}, 1 \mathrm{~mm}, 2 \mathrm{~mm}$ and $3 \mathrm{~mm}$, respectively, with the fluctuation of less than $1 \mathrm{~cm}$. At the same time, the maximum absolute value of measurement errors are $27 \mathrm{~mm}, 19 \mathrm{~mm}, 1 \mathrm{~mm}$ and $13 \mathrm{~mm}$, respectively. The measurement at $1600 \mathrm{~mm}$ is more accurate, which is consistent with the moderate distance and small error experimental results in the measurement range experiment. The experimental result proves that the overall system error is less than $3 \mathrm{~cm}$.

\section{Conclusions}

Based on the study of ultrasonic transmission path, this paper selects the ultrasonic transducer. The transformerless ultrasonic driving circuit was designed by calculating the energy of the critical energy storage components in normal and fault conditions. The parameter setting for implementing the wave-processing circuit based on LIN bus technology was completed, and the transmission scheme of the comparison result was demonstrated. Finally, the above-mentioned ultrasonic transceiver scheme was verified by the e524.06 chip, and the ultrasonic ranging system without a transformer was built to verify the ranging performance. The experiment proves that the ultrasonic ranging system can realize distance measurement in the range of $250-2700 \mathrm{~mm}$ with an error of less than $30 \mathrm{~mm}$ and a measurement fluctuation of less than $10 \mathrm{~mm}$, which provides an effective method for improving the anti-interference ability of the mining ultrasonic ranging system.

Author Contributions: Formal analysis, H.T.; investigation, D.W.; methodology, M.Z.; project administration, H.Z.; software, Y.G.; validation, Y.Q.; writing of the original draft, Y.W.; writing of review and editing, B.J.

Acknowledgments: This work was supported in part by the National Natural Science Foundation of China under grant No. 51504161. This work was supported in part by the Natural Science Foundation of Shanxi Province under grant No. 201701D221115. This work was supported in part by the Research Project Supported by Shanxi Scholarship Council of China under grant No. 2016-035.

Conflicts of Interest: The authors declare no conflict of interest.

\section{References}

1. Dash, A.K.; Bhattcharjee, R.M.; Paul, P.S.; Tikader, M. Study and Analysis of Accidents Due to Wheeled Trackless Transportation Machinery in Indian Coal Mines-Identification of Gap in Current Investigation System. Procedia Earth Planet. Sci. 2015, 11, 539-547. [CrossRef]

2. Kumar, S.; Furuhashi, H. Long-range measurement system using ultrasonic range sensor with high-power transmitter array in air. Ultrasonics 2017, 74, 186-195. [CrossRef] [PubMed] 
3. Yao, Z.; Hong, L.; Cheng, L. Improvement of measurement distance in multi-channel ultrasonic ranging systems through adaptive chaotic pulse position width modulation excitation sequences. Insight Non-Destr. Test. Cond. Monit. 2016, 58, 324-330. [CrossRef]

4. Mu, W.Y.; Zhang, G.P.; Huang, Y.M.; Yang, X.G.; Liu, H.Y.; Yan, W. Omni-Directional Scanning Localization Method of a Mobile Robot Based on Ultrasonic Sensors. Sensors 2016, 16, 2189. [CrossRef] [PubMed]

5. Gabriel, G. Indoor Pedestrian Navigation Using Foot-Mounted IMU and Portable Ultrasound Range Sensors. Sensors 2011, 11, 7606-7624.

6. Yao, Y.; Ju, X.; Lu, J.; Men, B. Acoustic Emission and Echo Signal Compensation Techniques Applied to an Ultrasonic Logging-While-Drilling Caliper. Sensors 2017, 17, 1351. [CrossRef] [PubMed]

7. Zhang, H.; Wang, Y.; Zhang, X.; Wang, D.; Jin, B. Design and Performance Analysis of an Intrinsically Safe Ultrasonic Ranging Sensor. Sensors 2016, 16, 867. [CrossRef] [PubMed]

8. CEN: Explosive Atmospheres e Explosion Prevention and Protection Part 1: Basic Conceptsand Methodology; EN 1127-01; European Committee For Standardization: Brussels, Belgium, 2011.

9. Davis, S.; Kelly, S.; Somandepalli, V. Hot Surface Ignition of Performance Fuels. Fire Technol. 2010, 46, 363-374. [CrossRef]

10. Simon, L.H.; Wilkens, V.; Beyer, M. Safety-related conclusions for the application of ultrasound in explosive atmospheres. J. Loss Prev. Process. Ind. 2015, 36, 522-531. [CrossRef]

11. Addai, E.K.; Gabel, D.; Krause, U. Experimental investigations of the minimum ignition energy and the minimum ignition temperature of inert and combustible dust cloud mixtures. J. Hazard. Mater. 2016, 307, 302-311. [CrossRef] [PubMed]

12. Zhang, Q.; Li, W.; Zhang, S. Effects of spark duration on minimum ignition energy for methane/air mixture. Process. Saf. Prog. 2011, 30, 154-156. [CrossRef]

13. Li, Y.; Zhu, H. A simple optimization method for the design of a lightweight, explosion-proof housing for a coal mine rescue robot. J. Braz. Soc. Mech. Sci. Eng. 2018, 40, 340. [CrossRef]

14. Solheim, F.; Arntzen, B.J.; Eckhoff, R.K. Effect of rusting and mechanical damage of gap surfaces on efficiency of flame gaps in flameproof electrical apparatus. Process. Saf. Environ. Prot. 2012, 90, 317-325. [CrossRef]

15. Jahdali, R.A.; Wu, Y. High transmission acoustic focusing by impedance-matched acoustic meta-surfaces. Appl. Phys. Lett. 2016, 108, 82-149. [CrossRef]

16. Toda, M.; Thompson, M. Novel multi-layer polymer-metal structures for use in ultrasonic transducer impedance matching and backing absorber applications. IEEE Trans. Ultrason. Ferroelectr. Freq. Control 2010, 57, 2818-2827. [CrossRef] [PubMed]

17. Kazys, R.J.; Sliteris, R.; Sestoke, J. Air-Coupled Ultrasonic Receivers with High Electromechanical Coupling PMN-32\%PT Strip-Like Piezoelectric Elements. Sensors 2017, 17, 2365. [CrossRef] [PubMed]

18. Kim, H.; Priya, S.; Stephanou, H.; Uchino, K. Consideration of impedance matching techniques for efficient piezoelectric energy harvesting. IEEE Trans. Ultrason. Ferroelectr. Freq. Control 2007, 54, 1851-1859. [CrossRef] [PubMed]

19. Terçariol, W.L.; Ferreira, E.C.; Dias, J.A.S. Analogue control of the slew-rate in LIN bus digital transitions using translinear circuits. Analog Integr. Circuits Signal Process. 2012, 72, 325-331. [CrossRef]

20. Fang, H.; Han, J.; Wei, Z. Modeling Method of Automotive Body CAN/LIN Nets Application Protocol Based on Object-oriented Colored Petri Net. Chin. J. Mech. Eng. 2011, 24, 999-1006. [CrossRef]

21. Wan, S.; Jia, M.; Li, L.; Duan, Y.; Sun, Y.; Zhang, W.; Zheng, X.; Gao, J.; Song, Y.; Shi, S.; et al. Ultrasonic Testing Technology and Application; China Machine Press: Beijing, China, 2017; p. 24.

22. Zhang, B. Safety Spark Circuit; China Coal Industry Publishing House: Beijing, China, 1981; p. 45.

23. IEC/SC31G: Explosive Atmospheres-Part 11: Equipment Protection by Intrinsic Safety "i"; IEC 60079-11-2006; International Electrotechnical Commission: Geneva, Switzerland, 2006.

24. Zhang, Y.; Li, W. Intrinsically Safe Circuit Design; China Coal Industry Publishing House: Beijing, China, 1991; p. 6.

(C) 2018 by the authors. Licensee MDPI, Basel, Switzerland. This article is an open access article distributed under the terms and conditions of the Creative Commons Attribution (CC BY) license (http:/ / creativecommons.org/licenses/by/4.0/). 